\title{
High Adherence to HIV Pre-Exposure Prophylaxis among Veterans
}

\author{
Misha Huang, MD, MS ${ }^{1,2}$, Wenhui Liu, MS $S^{2}$, Mary E. Plomondon, PhD ${ }^{2,3}$, \\ Allan V. Prochazka, MD, MSc ${ }^{2,3}$, and Mary T. Bessesen, $\mathrm{MD}^{1,2}$ \\ 'Division of Adult Infectious Diseases, University of Colorado Denver, Aurora, CO, USA; ${ }^{2}$ Department of Veterans Affairs Eastern Colorado \\ Healthcare System, Denver, CO, USA; ${ }^{3}$ University of Colorado Denver, Aurora, CO, USA.
}

KEY WORDS: HIV; AIDS; Adherence; Veterans.

J Gen Intern Med 33(3):253-5

DOI: $10.1007 / \mathrm{s} 11606-017-4260-2$

(c) Society of General Internal Medicine (outside the USA) 2018

\section{INTRODUCTION}

HIV pre-exposure prophylaxis (PrEP) with daily oral tenofovir disoproxil fumarate/emtricitabine (TDF/FTC) has been shown in clinical trials to be efficacious in preventing HIV infection in high-risk adults. ${ }^{1}$ Cases of failure and incident HIV infection in the major clinical trials were associated with poor medication adherence. ${ }^{2}$ Despite a large number of individuals who would benefit from PrEP use, concerns about poor medication adherence remain a barrier to wider dissemination of this intervention. ${ }^{3}$ Few studies have evaluated adherence to HIV PrEP in routine clinical settings. The Department of Veterans Affairs (VA) is the largest HIV care provider in the United States. PrEP is a covered benefit for all enrolled Veterans.

\section{METHODS}

This was a retrospective cohort study of Veterans who initiated PrEP between July 2012 and June 2016, using data extracted from the VA clinical and administrative databases. PrEP adherence was measured as the proportion of days covered (PDC). PDC was calculated by dividing the total number of non-hospitalized days during which TDF/FTC was supplied, by the number of days in the observation period. Though previous studies have demonstrated that high PrEP efficacy may be achievable with $60 \%$ adherence, ${ }^{4}$ standard adherence measures in other clinical settings using pharmacy refill data define adherence as $\geq 80 \%{ }^{5}$ In this study, we defined $<60 \%$ PDC as suboptimal, $60-79 \%$ PDC as acceptable, and $\geq 80 \%$ PDC as optimal adherence. Incident HIV infections were identified by screening records for HIV ICD-9 codes, ICD10 codes, and HIV polymerase chain reaction (PCR) results. Diagnoses were confirmed with manual chart review.

\section{RESULTS}

We identified 1074 patients who initiated PrEP between July 2012 and June 2016. Among these, 989 had more than one

Published online January 4, 2018 medication fill and were included in our final cohort for analysis (Fig. 1).

The mean age of a patient starting PrEP was 41.5 years (SD 12.37), and the majority of this cohort were men (96\%). Most initial PrEP prescriptions were prescribed by infectious disease specialists (72\%), though $22 \%$ of PrEP was initiated by a primary care provider; these were similar for both adherent and non-adherent patients. The mean PDC was $83 \%$ for the entire cohort (SD 21\%; Table 1).

We observed an increasing trend in PrEP adherence for each subsequent calendar year during which PrEP was initiated. In 2012, 75\% of patients initiating PrEP achieved protective levels of adherence ( $\geq 60 \%$ PDC); this proportion rose to $90 \%$ of patients who started PrEP in 2016 ( $p=0.004$; Fig. 1). Fifty-six percent of patients achieved optimal adherence in 2012, and this proportion rose to $76 \%$ of patients who started PrEP in 2016 ( $p=0.004)$.

In multivariable analysis, age, race, year of PrEP initiation, and number of other chronic medications were significantly associated with optimal adherence. Adherence increased by 9\% per year for new PrEP starts (adjusted relative risk [aRR] $1.09 ; 95 \%$ CI 1.03, 1.15; $p=0.001$ ). There were no differences in adherence between patients who were prescribed PrEP by an infectious disease provider versus primary care or other providers, between patients who initiated PrEP at a community-based outpatient clinic/health care center (CBOC/HCC) versus a VAMC, or between patients who lived in rural/highly rural areas versus urban areas. However, black race remained significantly associated with less than optimal $(<80 \%)$ adherence, even after adjusting for covariates (aRR 0.76 ; $95 \%$ CI $0.66,0.86 ; p<0.001$ ).

Six incident HIV infections were observed among our cohort. Three patients were diagnosed with HIV while on PrEP ( $0.3 \%$ of the cohort), and three were diagnosed after PrEP discontinuation. All three patients who seroconverted while on PrEP were diagnosed at their first 3-month follow-up. One reported missing several days of medication prior to an HIV exposure, and all three had M184V mutations (conferring resistance to FTC) on subsequent genotype testing.

\section{DISCUSSION}

PrEP adherence within the VA has increased in recent years, and current rates of adherence are high, with $90 \%$ of $\mathrm{PrEP}$ 


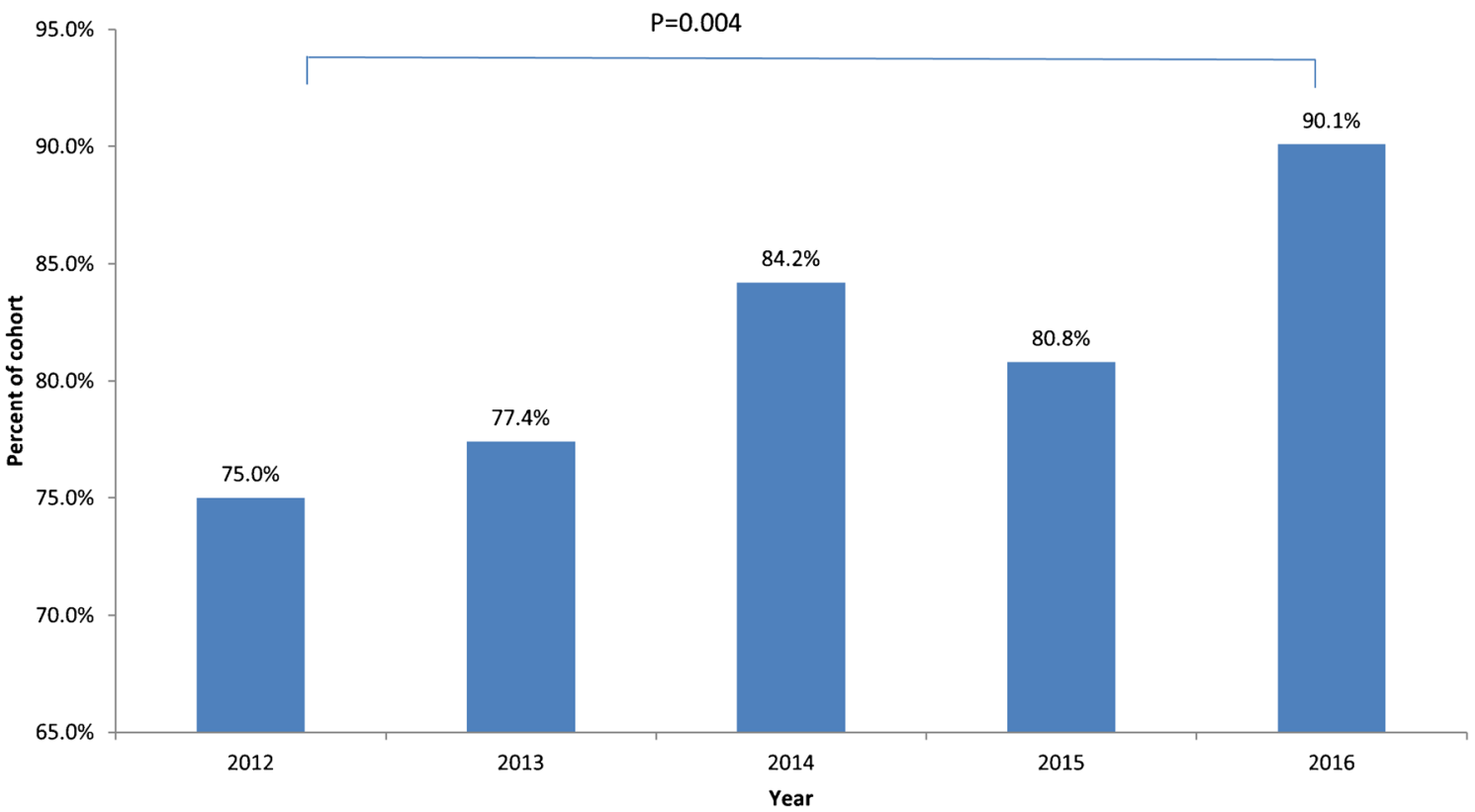

Figure 1 Change in percentage of VA PrEP users with protective levels (acceptable or optimal) of adherence ( $\geq 60 \%$ PDC) over time.

users achieving protective levels. However, black patients, who are already disproportionately affected by HIV, remain at risk for less than optimal adherence. Simplification of medication regimens in general may help promote better PrEP adherence. Few cases of HIV seroconversion occurred in patients while on PrEP. Overall, real-world adherence to PrEP has improved over time, and the vast majority of patients are at acceptable levels.

Table 1 Demographic Characteristics of PrEP Users in the VA

\begin{tabular}{|c|c|c|c|c|}
\hline Characteristic* & $\begin{array}{l}\text { Entire cohort } \\
N=989\end{array}$ & $\begin{array}{l}\text { Optimal adherence } \\
(\geq 80 \% \text { PDC) } \\
n=674(68.15 \%)\end{array}$ & $\begin{array}{l}\text { Acceptable or suboptimal } \\
\text { adherence }(<80 \% \text { PDC) } \\
n=315\end{array}$ & $p$ value \\
\hline Age, mean (SD), years & $41.49(12.37)$ & $42.39(12.40)$ & $39.58(12.11)$ & $<0.001$ \\
\hline Sex, no. $(\%)$ & & & & 0.75 \\
\hline Male & $951(96.16)$ & $649(96.29)$ & $302(95.87)$ & \\
\hline Female & $38(3.84)$ & $25(3.71)$ & $13(4.13)$ & \\
\hline Race, no. (\%) & & & & $<0.001$ \\
\hline White & $613(61.98)$ & $444(65.88)$ & $169(53.65)$ & \\
\hline Black & $225(22.75)$ & $122(18.10)$ & $103(32.70)$ & \\
\hline Other & $151(15.27)$ & $108(16.02)$ & $43(13.65)$ & \\
\hline Ethnicity, no. (\%) & & & & 0.59 \\
\hline Hispanic & $132(13.35)$ & $85(12.71)$ & $47(14.92)$ & \\
\hline Non-Hispanic & $797(80.59)$ & $545(81.46)$ & $252(80.00)$ & \\
\hline Unknown & $60(6.06)$ & $44(5.83)$ & $16(5.08)$ & \\
\hline Rurality, no. (\%) & & & & 0.29 \\
\hline Urban & 887 (89.69) & $598(88.86)$ & $289(92.04)$ & \\
\hline Rural/Highly rural & $100(10.11)$ & $75(11.14)$ & $25(7.96)$ & \\
\hline Provider specialty, no. (\%) & & & & 0.31 \\
\hline Infectious disease & $712(71.99)$ & $489(72.55)$ & $223(70.79)$ & \\
\hline Primary care & $222(22.45)$ & $149(22.11)$ & $73(23.17)$ & \\
\hline Other & $55(5.56)$ & $36(5.34)$ & $19(6.03)$ & \\
\hline VA facility type, no. (\%) & & & & 0.11 \\
\hline $\mathrm{CBOC} / \mathrm{HCC}$ & 115 (11.94) & $77(11.74)$ & $38(12.38)$ & \\
\hline VAMC & $848(88.06)$ & $579(88.26)$ & $269(87.62)$ & \\
\hline Number of other chronic medications, no. (\%) & & & & 0.02 \\
\hline $1-4$ & $679(68.66)$ & $448(66.47)$ & $231(73.33)$ & \\
\hline$\geq 5$ & $310(31.34)$ & $226(33.53)$ & $84(26.67)$ & \\
\hline Proportion of days covered, mean (SD) & $0.83(0.21)$ & $0.95(0.06)$ & $0.57(0.19)$ & $<0.001$ \\
\hline
\end{tabular}

*Data analyzed were at the time of the index TDF/FTC prescription (i.e., at the time of PrEP initiation)

PrEP pre-exposure prophylaxis, PDC proportion of days covered, CBOC/HCC community-based outpatient clinic/health care center, VAMC VA Medical Center 
Corresponding Author: Mary T. Bessesen, MD; Division of Adult Infectious DiseasesUniversity of Colorado Denver, Aurora, CO, USA (e-mail: Mary.Bessesen@ucdenver.edu).

\section{Contributors None.}

Funders This study was supported by the Department of Veterans Affairs, Veterans Health Administration, Office of Research and Development, Health Services Research and Development Fellowship Program, the Denver-Seattle Center of Innovation, and the Colorado Clinical and Translational Sciences Institute at the University of Colorado. The funding source had no role in the design or conduct of the study; collection, management, analysis, or interpretation of the data; preparation, review, or approval of the manuscript; or the decision to submit the manuscript for publication. The content of this manuscript is solely the responsibility of the authors, and does not necessarily reflect the views or policies of the Department of Veterans Affairs or the United States Government.

\section{Compliance with Ethical Standards:}

Prior Presentations: None.
Conflict of Interest: All authors declare that they do not have a conflict of interest.

\section{REFERENCES}

1. Grant RM, Lama JR, Anderson PL, et al. Preexposure chemoprophylaxis for HIV prevention in men who have sex with men. N Engl J Med. 2010;363(27):2587-99.

2. Koenig LJ, Lyles C, Smith DK. Adherence to antiretroviral medications for HIV pre-exposure prophylaxis: lessons learned from trials and treatment studies. Am J Prev Med. 2013;44(1:Suppl 2):S91-8.

3. Smith DK, Van HM, Wolitski RJ, et al. Vital Signs: Estimated Percentages and Numbers of Adults with Indications for Preexposure Prophylaxis to Prevent HIV Acquisition-United States, 2015. MMWR Morb Mortal Wkly Rep. 2015;64(46):1291-5.

4. Anderson PL, Glidden DV, Liu A, et al. Emtricitabine-tenofovir concentrations and pre-exposure prophylaxis efficacy in men who have sex with men. Sci Transl Med. 2012;4(151):151ra125.

5. Hess LM, Raebel MA, Conner DA, Malone DC. Measurement of adherence in pharmacy administrative databases: a proposal for standard definitions and preferred measures. Ann Pharmacother. 2006;40(78):1280-8. 